\title{
HAAR MEASURE FOR COMPACT RIGHT TOPOLOGICAL GROUPS
}

\author{
PAUL MILNES AND JOHN PYM
}

(Communicated by Jonathan M. Rosenberg)

\begin{abstract}
Compact right topological groups arise in topological dynamics and in other settings. Following H. Furstenberg's seminal work on distal flows, R. Ellis and I. Namioka have shown that the compact right topological groups of dynamical type always admit a probability measure invariant under the continuous left translations; however, this invariance property is insufficient to identify a unique probability measure (in contrast to the case of compact topological groups). In the present paper, we amplify on the proofs of Ellis and Namioka to show that a right invariant probability measure on the compact right topological group $G$ exists provided $G$ admits an appropriate system of normal subgroups, that it is uniquely determined and that it is also invariant under the continuous left translations. Using Namioka's work, we show that $G$ has such a system of subgroups if its topological centre contains a countable dense subset, or if it is a closed subgroup of such a group.
\end{abstract}

\section{Preliminaries}

For notation and terminology we will follow Namioka [9] and, especially, Berglund et al. [4] as much as possible. Thus a (compact) right topological group is a pair $(G, \tau)$, where $G$ is a group and $\tau$ is a (compact) topology on $G$, so that the right translations $t \mapsto t s, G \rightarrow G$ are continuous for all $s \in G$. The topological centre $\Lambda(G)$ of $G$ is the set

$$
\{s \in G \mid t \mapsto s t, G \rightarrow G \text { is continuous }\} .
$$

If $\Lambda(G)=G$, then $G$ is called semitopological; if $G$ is also Hausdorff, then $G$ is a topological group, by Ellis's famous theorem [5]. A probability measure $\mu$ on $G$ is called left invariant if it is invariant under the continuous left translations, i.e., if $\mu(s B)=\mu(B)$ for all $s \in \Lambda(G)$ and all Borel sets $B \subset G$. (It follows from a remark at the end of [2] that a compact right topological group need not have a unique left invariant probability measure.) We call $\mu$ right invariant and a Haar measure if it is invariant under all right translations, i.e., if $\mu(B s)=\mu(B)$ for all $s \in G$ and all Borel sets $B \subset G$.

Received by the editors July 5, 1990 and, in revised form, August 2, 1990.

1980 Mathematics Subject Classification (1985 Revision). Primary 43A05, 22 C05.

Key words and phrases. Compact right topological group, strong normal system of subgroups, invariant probability measure.

This research was supported in part by NSERC grant A7857. 
For a compact, Hausdorff, right topological group $(G, \tau)$ with $\Lambda(G)$ dense in $G$, the $\sigma$-topology of $G$ is determined by taking

$$
\mathscr{U}:=\left\{U^{-1} U \mid U \text { is an open } \tau \text {-neighbourhood of the identity } e \in G\right\}
$$

to be a base of $\sigma$-neighbourhoods of $e$. Then $\sigma$ is compact, $T_{1}$, and coarser than $\tau ;(G, \sigma)$ is a compact, $T_{1}$, semitopological group with continuous inversion. ( $\sigma$ is to suggest "symmetrized.") But $\sigma$ is not usually Hausdorff; indeed, it is Hausdorff if and only if it is the same as $\tau$, and then $(G, \tau)$ is a topological group. If $L$ is a subgroup of $G$, the quotient space $G / L$ is Hausdorff in the quotient topology of $\tau$ if and only if $L$ is $\sigma$-closed. See Namioka [9; §1] (or Berglund et al. [4; Appendix C]) for the details.

A most important concept for us is that of a strong normal system $\left\{L_{\xi} \mid \xi \leq\right.$ $\left.\xi_{0}\right\}$ of subgroups of a compact, Hausdorff right topological group $(G, \tau)$. The system is indexed by the set of ordinals less than or equal to an ordinal $\xi_{0}$ and satisfies

(i) each $L_{\xi}$ is a $\sigma$-closed normal subgroup of $G, L_{0}=G$ and $L_{\xi_{0}}=\{e\}$;

(ii) for $\xi<\xi_{0}, L_{\xi} \subset L_{\xi+1}$ and the function

$$
\left(s L_{\xi+1}, t L_{\xi+1}\right) \mapsto s t L_{\xi+1}, G / L_{\xi+1} \times L_{\xi} / L_{\xi+1} \rightarrow G / L_{\xi+1}
$$

is continuous for the quotient topologies from $\tau$; and

(iii) for each limit ordinal $\xi \leq \xi_{0}, L_{\xi}=\bigcap_{\eta<\xi} L_{\eta}$.

It follows that each $G / L_{\xi}$ is a group and, in the quotient topology of $\tau$, is compact, Hausdorff, and right topological with $\Lambda(G) / L_{\xi} \subset \Lambda\left(G / L_{\xi}\right.$ ). (See 1.3.7-8 in Berglund et al. [4], for example.) Hence, each $L_{\xi} / L_{\xi+1}$ is a compact, Hausdorff, topological group.

\section{Existence AND UNiQueness of HAar MeAsure}

Our aim is to prove

Theorem. Let $(G, \tau)$ be a compact, Hausdorff, right topological group that has a strong normal system of subgroups $\left\{L_{\xi} \mid \xi \leq \xi_{0}\right\}$. Then Haar measure exists on $G$. It is the unique right invariant probability measure on $G$; it is also left invariant.

Proof. We prove this theorem by referring to proofs in Namioka [9] (or in Berglund et al. [4]), but owing to the complexity of these proofs we need to be clear about how the changes we require are made.

Haar measure for $G$ is constructed by transfinite induction. It will appear as a positive, invariant, linear functional of norm 1 on $\mathscr{C}(G)$. Given $L:=L_{\xi}$ and $M:=L_{\xi+1}$ in the strong normal system, let $\mathscr{C}_{L}(G)$ be the subspace of $\mathscr{C}(G)$ consisting of functions constant on the cosets of $L$. Let $\nu_{L}$ be Haar measure on the compact, Hausdorff, topological group $L / M$, and for $s \in G$, let $\bar{s}$ denote the coset $s M=M s \in G / M$. Each $f \in \mathscr{C}_{M}(G)$ gives rise to a function $\bar{f} \in \mathscr{C}(G / M), \bar{f}(\bar{s})=f(s)$. The continuity of $G / M \times L / M \rightarrow G / M$ shows that for $f \in \mathscr{C}_{M}(G)$ the function $\varphi_{L}(f)$ defined by

$$
\varphi_{L}(f)(s)=\int_{L / M} \bar{f}(\bar{s} \bar{t}) d \nu_{L}(\bar{t}) \quad(s \in G)
$$

is continuous. Since $\nu_{L}$ is Haar measure on $L / M, \varphi_{L}(f)$ is constant on the cosets of $L$. Thus $\varphi_{L}$ maps $\mathscr{C}_{M}(G)$ into $\mathscr{C}_{L}(G)$. It is easily seen that $\varphi_{L}$ 
is a retraction (i.e., $\mathscr{C}_{M}(G) \subset \mathscr{C}_{L}(G)$ and $\varphi_{L}$ is the identity on $\mathscr{C}_{L}(G)$ ). This part of the proof (the induction step for successor ordinals) is Lemma 4.1 in Namioka [9] (Lemma C.16 in Berglund et al. [4]).

We next check that right translation by elements of $G$ commutes with $\varphi_{L}$, i.e., that $R_{u} \varphi_{L}=\varphi_{L} R_{u}$, where $\left(R_{u} f\right)(s)=f(s u)$ for $u, s \in G$ and $f \in \mathscr{C}(G)$. First, observe that for fixed $u \in G$ the maps

$$
\bar{t} \mapsto \bar{u} \bar{t} \mapsto \bar{u} \bar{t} \bar{u}^{-1}, L / M \rightarrow G / M \rightarrow L / M \subset G / M
$$

are continuous, so that

$$
g \mapsto \int_{L / M} g\left(\bar{u} \bar{u} \bar{u}^{-1}\right) d \nu_{L}(\bar{t})
$$

is a positive, normalized, invariant, linear functional on $\mathscr{C}(L / M)$, hence is also Haar measure $\nu_{L}$. Therefore

$$
\begin{aligned}
\left(R_{u} \varphi_{L}(f)\right)(s) & =\varphi_{L}(f)(s u)=\int_{L / M} \bar{f}(\overline{s u} \bar{t}) d \nu_{L}(\bar{t}) \\
& =\int_{L / M} \bar{f}\left(\bar{s} \bar{u} \bar{t} \bar{u}^{-1} \bar{u}\right) d \nu_{L}(\bar{t})=\int_{L / M} \bar{f}(\bar{s} \bar{u} \bar{u}) d \nu_{L}(\bar{t}) \\
& =\int_{L / M}\left(\overline{R_{u} f}\right)(\bar{s} \bar{t}) d \nu_{L}(\bar{t})=\varphi_{L}\left(R_{u} f\right)(s) .
\end{aligned}
$$

To fit the last two paragraphs into a transfinite induction setting, observe that $\mathscr{C}_{L_{0}}(G)=\mathbb{C}$. If we have already determined the appropriate linear map

$$
\psi_{\xi-1}: \mathscr{C}_{L_{\xi-1}}(G) \rightarrow \mathscr{C}_{L_{0}}(G)=\mathbb{C},
$$

we define $\psi_{\xi}=\varphi_{L_{\xi}} \circ \psi_{\xi-1}$. Then $\psi_{\xi}$ is positive, linear, of norm 1, and commutes with right translations.

Now we come to limit ordinals. Suppose that $\psi_{\eta}$ is defined for all $\eta$ less than the limit ordinal $\xi$. Observe that $\bigcup_{\eta<\xi} \mathscr{C}_{L_{\eta}}(G)$ is a subalgebra of $\mathscr{C}_{L_{\xi}}(G)$ containing the constants. If $x, y \in G$ and $x L_{\xi} \neq y L_{\xi}$, then $y \notin x L_{\xi}=$ $\bigcap_{\eta<\xi} x L_{\eta}$. Thus there is an $\eta$ with $y \notin x L_{\eta}$, and so there is an $f \in \mathscr{C}_{L_{\eta}}(G)$ with $f(x) \neq f(y)$. It follows from a version of the Stone-Weierstrass theorem that $\bigcup_{\eta<\xi} \mathscr{C}_{L_{\eta}}(G)$ is dense in $\mathscr{C}_{L_{\xi}}(G)$. It is now obvious how to define $\psi_{\xi}$ : $\mathscr{C}_{L_{\xi}}(G) \rightarrow \mathbb{C}$, a positive linear functional of norm 1 commuting with right translations.

Obviously, $\mathscr{C}_{L_{\xi_{0}}}(G)=\mathscr{C}(G)$. So $\psi_{\xi_{0}}$ is a Haar measure on $G$.

Uniqueness is also proved by transfinite induction. Let $\mu$ be a right invariant measure on $G$. We show that

$$
\int_{G} f d \mu=\psi_{\xi}(f) \quad\left(f \in \mathscr{C}_{L_{\xi}}(G)\right) .
$$

This holds for $\xi=0$. Suppose it holds for $\xi$. Take $f \in \mathscr{C}_{L_{\xi+1}}(G)$. Let $\mu_{\xi}$ denote the projection of $\mu$ on $G / L_{\xi+1}$. Then

$$
\begin{aligned}
\int_{G} f d \mu & =\int_{G / L_{\xi+1}} \bar{f}(\bar{s}) d \mu_{\xi}(\bar{s})=\int_{G / L_{\xi+1}} \int_{L_{\xi} / L_{\xi+1}} \bar{f}(\bar{s}) d \nu_{L_{\xi}}(\bar{t}) d \mu_{\xi}(\bar{s}) \\
& =\int_{G / L_{\xi+1}} \int_{L_{\xi} / L_{\xi+1}} \bar{f}(\bar{s} \bar{t}) d \nu_{L_{\xi}}(\bar{t}) d \mu_{\xi}(\bar{s})=\int_{G / L_{\xi+1}} \overline{\varphi_{L_{\xi}}(f)}(\bar{s}) d \mu_{\xi}(\bar{s}) \\
& =\int_{G} \varphi_{L_{\xi}}(f) d \mu=\psi_{\xi}\left(\varphi_{L_{\xi}}(f)\right)=\psi_{\xi+1}(f),
\end{aligned}
$$


as required (where we have used Fubini's theorem and right invariance of $\mu$ to get the third equality). Limit ordinals are again treated using the StoneWeierstrass theorem. Thus $\mu$ corresponds to the linear functional $\psi_{\xi_{0}}$. Uniqueness follows.

If $f \in \mathscr{C}(G)$ and $t \in \Lambda(G)$, then $s \mapsto f(t s)$ is continuous on $G$. Therefore, if $\mu$ is right invariant, then also $f \mapsto \int_{G} f(t s) d \mu(s)$ is well-defined and right invariant, so that it is just $\mu$. We have proved left invariance.

We next identify a large class of compact right topological groups having strong normal systems of subgroups.

The Furstenberg-Ellis-Namioka Structure Theorem. Let $\left(G_{1}, \tau\right)$ be a compact, Hausdorff, right topological group whose topological centre $\Lambda\left(G_{1}\right)$ contains a countable subset dense in $G_{1}$. Let $G$ be a closed subgroup of $G_{1}$. Then $G$ has a strong normal system of subgroups.

Proof. This theorem is a translation of the Furstenberg structure theorem for distal flows [8] into the context of compact right topological groups. It is stronger than the currently accepted form of this translation (Namioka [9; Corollary 2.1] or Berglund et al. [4]) in two ways. First, it asserts that the result holds for closed subgroups $G$ of $G_{1}$ and not just for $G_{1}$ itself. Secondly, it claims that each group $L_{\xi}$ is normal in $G$ and not simply that each $L_{\xi+1}$ is normal in $L_{\xi}$.

Let us deal with the first difference. If the existence of a system $\left\{L_{\xi}\right\}$ of normal subgroups is established for $G_{1}$, we simply form the system $\left\{G \cap L_{\xi}\right\}$ to obtain a system for $G$. These subgroups are closed in the $\sigma$-topology of $G$ because they are closed in the restriction to $G$ of the $\sigma$-topology of $G_{1}$, the latter topology being weaker than the former. (It is conceivable that $\Lambda(G)$ is not dense in $G$, so it is necessary here to go back to the basic definition of the $\sigma$-topology; see (ii) of Remark 1 below.)

We shall therefore assume that $G_{1}=G$ in what follows. As stated in the preliminaries, when $\Lambda(G)$ is dense in $G$ the $\sigma$-topology on $G$ is determined by taking

$$
\mathscr{U}:=\left\{U^{-1} U \mid U \text { is an open } \tau \text {-neighbourhood of the identity } e \in G\right\}
$$

to be a base of $\sigma$-neighbourhoods of $e$. We give another description of $\mathscr{U}$, namely

$$
\mathscr{U}=\mathscr{U}_{1}:=\left\{V^{-1} V \mid V \neq \phi \text { is } \tau \text {-open in } G\right\} .
$$

Clearly, $\mathscr{U} \subset \mathscr{U}_{1}$. On the other hand, if $V \neq \phi$ is $\tau$-open, there is a point $t \in V \cap \Lambda(G)$. Then $t^{-1} V$ is a neighbourhood of the identity, and

$$
V^{-1} V=\left(t^{-1} V\right)^{-1}\left(t^{-1} V\right) \in \mathscr{U}
$$

We digress to do some analysis on a compact semitopological group $(H, \omega)$ (as in Namioka [9; p. 196] or Berglund et al. [4; Appendix C]). Define

$$
N=N(H):=\bigcap\left\{W_{0} \mid W_{0} \text { is a closed neighbourhood of } e \in H\right\} .
$$

Then the following statements about $s \in H$ are easily seen to be equivalent:

(a) $s \in N$;

(b) for every neighbourhood $W$ of $e, s \in W^{-}$;

(c) for every pair of neighbourhoods $W$ and $W_{1}$ of $e, W_{1} s \cap W \neq \phi$;

(d) for every neighbourhood $W$ of $e, W s \cap W \neq \phi$; and

(e) for every neighbourhood $W$ of $e, s \in W^{-1} W$. 
Thus $N=\bigcap\left\{W^{-1} W \mid W\right.$ is a neighbourhood of $\left.e\right\}$, i.e., $N$ is the closure of $\{e\}$ in the $\sigma$-topology of $H$, in which $H$ is a compact semitopological group with continuous inversion; so $N$, the closure in this topology of the normal subgroup $\{e\}$, is also a normal subgroup of $H$.

We apply the analysis of the last paragraph to a $\sigma$-closed subgroup $L$ of $G$ furnished with the restriction of the $\sigma$-topology of $G$. For simplicity, we refer to this topology on $L$ also as $\sigma ;(L, \sigma)$ is a compact, $T_{1}$, semitopological group. For $(L, \sigma)$

$$
\begin{aligned}
N & =N(L)=\bigcap\left\{W_{0} \mid W_{0} \text { is a } \sigma \text {-closed } \sigma \text {-neighbourhood in } L \text { of } e\right\} \\
& =\bigcap\left\{W^{-1} W \mid W \text { is a } \sigma \text {-neighbourhood in } L \text { of } e\right\} .
\end{aligned}
$$

A basis for $\sigma$-neighbourhoods in $L$ of $e$ is

$$
\left\{U^{-1} U \cap L \mid U \text { is an open } \tau \text {-neighbourhood in } G \text { of } e\right\},
$$

which equals

$$
\left\{V^{-1} V \cap L \mid V \neq \phi \text { is } \tau \text {-open in } G\right\}
$$

(since $\mathscr{U}=\mathscr{U}_{1}$, as noted two paragraphs above). We observe that all these sets are symmetric, e.g., $\left(V^{-1} V \cap L\right)^{-1}=V^{-1} V \cap L$, and conclude that

$$
N(L)=\bigcap\left\{\left(V^{-1} V \cap L\right)\left(V^{-1} V \cap L\right) \mid V \neq \phi \text { is } \tau \text {-open in } G\right\} .
$$

The next step is to show that if, in addition, $L$ is normal in $G$, then so is $N(L)$. Fix $s \in G$. Note that $V s$ runs through all $\tau$-open subsets of $G$ when $V$ does and also that $s^{-1} L s=L$. Therefore, intersecting over all nonvoid $\tau$-open subsets of $G$, we get

$$
\begin{aligned}
N(L) & =\bigcap_{V}\left((V s)^{-1}(V s) \cap L\right)\left((V s)^{-1}(V s) \cap L\right) \\
& =\bigcap_{V}\left(s^{-1} V^{-1} V s \cap s^{-1} L s\right)\left(s^{-1} V^{-1} V s \cap s^{-1} L s\right) \\
& =s^{-1} \bigcap_{V}\left(V^{-1} V \cap L\right)\left(V^{-1} V \cap L\right) s=s^{-1} N(L) s .
\end{aligned}
$$

The family $\left\{L_{\xi}\right\}$ is produced by transfinite induction. Start with $L_{0}=G$. If $\xi$ has predecessor $\xi-1$, put $L_{\xi}=N\left(L_{\xi-1}\right)$. If $\xi$ is a limit ordinal, use (iii). Clearly, the $L_{\xi}$ 's are normal in $G$. A key point is that, if $L_{\xi} \neq\{e\}$, then $N\left(L_{\xi}\right) \neq L_{\xi}$; for this see Namioka [9; Theorem 2.3] (or Berglund et al. [4]). This last fact ensures that the induction process finishes at least by card $(G)$.

The continuity claim in (ii) is Proposition 2.1 in Namioka [9] (Lemma C.11 in Berglund et al. [4]), which asserts that

$$
(s N(L), t N(L)) \mapsto \operatorname{st} N(L), G / N(L) \times L / N(L) \rightarrow G / N(L)
$$

is continuous (for the quotient topologies from $\tau$ ). The structure theorem has been established.

Remarks. 1. The existence of a strong normal system of subgroups is not a necessary condition for the existence of Haar measure on a compact right topological group. Here is an example. (Other examples like this, which can be also connected and metrizable, are due to W. Ruppert [3; V.1.5].) 
Let $G$ be the semidirect product $\{ \pm 1\} \times \mathbb{T}$ with multiplication

$$
(\epsilon, u)(\delta, v)=\left(\epsilon \delta, u^{\delta} v\right) \text {. }
$$

Give $G$ the topology $\tau$ for which a typical basic neighbourhood of $\left(1, e^{i a}\right)$ or $\left(-1, e^{i b}\right)$, where $a<b$, is

$$
A:=\left\{\left(1, e^{i a}\right),\left(-1, e^{i b}\right)\right\} \cup\left\{\left(\epsilon, e^{i \theta}\right) \mid \epsilon= \pm 1, a<\theta<b\right\} ;
$$

these basic neighbourhoods are open and closed. Note that every open set $O$ is the union of a countable number of sets of form $A$. (The argument for this claim goes hardly beyond that required for the analogous claim about open sets of real numbers.) If we identify $\mathbb{T}_{1}:=\{1\} \times \mathbb{T}$ and $\mathbb{T}_{2}:=\{-1\} \times \mathbb{T}$ with $\mathbb{T}$ in the obvious way, it follows that the symmetric difference $\left(O \cap \mathbb{T}_{1}\right) \triangle\left(O \cap \mathbb{T}_{2}\right)$ is countable. Also, $\mathbb{T}_{i}$ is dense in $G, i=1,2$.

$(G, \tau)$ is a compact, Hausdorff, right topological group and $\Lambda(G)=\{(1,1)\}$. Since

$$
(\epsilon, u)(\delta, v)(\epsilon, u)^{-1}=\left(\epsilon \delta, u^{\delta} v\right)\left(\epsilon, u^{-\epsilon}\right)=\left(\delta, u^{\epsilon \delta-\epsilon} v\right),
$$

we see that a normal subgroup $L$ of $G$ contains $\mathbb{T}_{2}$ if it contains one member of $\mathbb{T}_{2}$, so $L=G$ if it is also closed. Thus the only nontrivial, closed, normal subgroups of $G$ are finite cyclic subgroups $H \subset \mathbb{T}_{1}$; the quotient of $G$ by such an $H$ is not a topological group, as it is isomorphic to $G$ (just as $\mathbb{T}$ modulo a finite subgroup is isomorphic to $\mathbb{T}$ ). We conclude that $G$ does not have a strong normal system of subgroups.

Nonetheless, $G$ has a unique Haar measure. This is so because any right invariant probability measure $\mu$ on $G$ must assign measure $\min \{1,(b-a) / 2 \pi\}$ to the basic neighbourhood $A$, so $\mu(B)=\eta\left(B \cap \mathbb{T}_{1}\right)$ for each Borel set $B \subset G$, where $\eta$ is (normalized) Lebesgue measure on $\mathbb{T}_{1} \cong \mathbb{T}$. The equation in the last line can be used to define Haar measure on $G$ in terms of $\eta$.

We mention some further calculations with this group that the interested reader can perform.

(i) A left translation $s \mapsto t s, G \rightarrow G$ is not measurable unless $t=( \pm 1,1)$. (Use the description of open sets given above.) The left translation $s \mapsto$ $(-1,1) s$ is measurable, but not continuous.

(ii) Since $\Lambda(G)$ is not dense in $G$, we must go back to the basic definition to discuss the $\sigma$-topology of $G$ : it is the quotient topology on $G$ of the product topology $\tau \times \tau$ on $G \times G$ under the map $(s, t) \mapsto s^{-1} t, G \times G \rightarrow G[9,4]$. In $G$, every $\sigma$-neighbourhood of $e$ is $\tau$-dense in $G$. Thus, the normal subgroup

$$
N(G)=\bigcap\left\{W_{0} \mid W_{0} \text { is a } \sigma \text {-closed } \sigma \text {-neighbourhood of } e\right\}
$$

(as in the proof of the structure theorem) is equal to $G$, an outcome that is not surprising in view of the description we have already given of the closed normal subgroups of $G$.

(iii) For this group, $\bigcap\left\{V^{-1} V V^{-1} V \mid V \neq \phi\right.$ is $\tau$-open in $\left.G\right\}$ (as in the proof of the structure theorem) turns out to be the nonnormal subgroup $\{( \pm 1,1)\}$.

2. Furstenberg [8] (for compact metrizable phase spaces) and Ellis [6, p. 164] (for any compact phase space) have produced invariant probability measures for minimal distal flows. This implies the existence of a left invariant probability measure on any compact right topological group $G$ with dense topological centre $\Lambda(G)$, since the (left) action of $\Lambda(G)$ on $G$ is distal-a conclusion also 
obtained by Namioka [9]. Our proofs have been on the lines followed by these authors. In particular, they first established structure theorems and the existence of invariant probability measures under countability hypotheses; Furstenberg and Ellis used metrizability, and Namioka used separability of $\Lambda(G)$ (which is the condition we have adopted). A compactness argument then made it easy for them to produce a left invariant probability measure on any compact right topological group [6, p. $164 ; 9$, p. 208]. Such an easy method of extending the result is not available to us, which is why we have complete presentation of our results only for groups $G$ with dense separable $\Lambda(G)$. However, the Furstenberg structure theorem for distal flows does not depend on any countability hypothesis; see Ellis [7] or Auslander [1]. This is also true of our Furstenberg-Ellis-Namioka structure theorem for compact right topological groups. The proof of this that we have at present is difficult, depending as it does on a detailed analysis of the proofs in Ellis's book. We hope to find a simpler version which we shall be able to publish later. But we should like to point out here that the main theorem of the present paper will then imply the existence of Haar measure for closed subgroups of any compact right topological group with dense topological centre.

3. We have not been able to characterise those compact right topological groups which have a strong normal system of subgroups; in particular, we do not know whether every such group must have a dense topological centre.

4. We expect that the fact that a compact right topological group $G$ with $\Lambda(G)$ dense in $G$ has a strong normal system of subgroups and Haar measure will have interesting consequences in topological dynamics and in other areas of analysis. We hope to develop some of them.

\section{BIBLIOGRAPHY}

1. J. Auslander, Minimal flows and their extensions, North-Holland, Amsterdam, 1988.

2. L. Auslander and F. Hahn, Real functions coming from flows on compact spaces and concepts of almost periodicity, Trans. Amer. Math. Soc. 106 (1963), 415-426.

3. J. F. Berglund, H. D. Junghenn, and P. Milnes, Compact right topological semigroups and generalizations of almost periodicity, Lecture Notes in Math., vol. 663, Springer-Verlag, New York, 1978.

4. __ Analysis on semigroups: function spaces, compactifactions, representations, Wiley, New York, 1989.

5. R. Ellis, Locally compact transformation groups, Duke Math. J. 24 (1957), 119-126.

6. __ Lectures on topological dynamics, Benjamin, New York, 1969.

7. __ The Furstenberg structure theorem, Pacific J. Math. 76 (1978), 345-349.

8. H. Furstenberg, The structure of distal flows, Amer. J. Math. 85 (1963), 477-515.

9. I. Namioka, Right topological groups, distal flows and a fixed point theorem, Math. Systems Theory 6 (1972), 193-209.

Department of Mathematics, University of Western Ontario, London, Ontario N6A 5B7, CANADA

Department of Mathematics, University of Sheffield, Sheffield S3 7RH, England 